\title{
USUÁRIOS DE UM CENTRO DE ATENÇÃO PSICOSSOCIAL: UM ESTUDO DE SUAS REPRESENTAÇÕES SOCIAIS ACERCA DE TRATAMENTO PSIQUIÁTRICO
}

Rubiane Rodrigues Mostazo ${ }^{1}$ Débora Isane Ratner Kirschbaum²

Mostazo RR, Kirschbaum DIR. Usuários de um centro de atenção psicossocial: um estudo de suas representações sociais acerca de tratamento psiquiátrico. Rev Latino-am Enfermagem 2003 novembro-dezembro; 11(6):786-91.

O objetivo do presente estudo é identificar e analisar as representações que usuários de um centro de atenção psicossocial constroem acerca do fenômeno tratamento psiquiátrico. Foram entrevistados 11 usuários do Centro de Atenção Psicossocial-CAPS/Estação. Os dados foram submetidos à análise de conteúdo, segundo Bardin, tendo como suporte referencial teórico-metodológico a teoria moscoviciana de representação social. Os temas, definidos a partir da análise dos dados, possibilitaram a estruturação de três categorias. Na primeira delas, tratar é ser medicado, estão contidos temas como o remédio como tratamento psiquiátrico, os efeitos do tratamento, os diferentes profissionais e sua participação no tratamento. A segunda categoria, tratar é ser cuidado/(des)cuidado, os temas apresentados foram: o cuidado no tratamento psiquiátrico, violência e instituição fechada. A terceira categoria, tratar é estar em atividade, foi constituída a partir dos temas: o tratamento e a atividade ocupacional, o tratamento psiquiátrico e o trabalho. Conclui-se que as representações de tratamento psiquiátrico estão fortemente ligadas ao uso do medicamento e ao médico, como precursor do tratamento.

DESCRITORES: saúde mental; psicologia social; enfermagem; desinstitucionalização

\section{USERS OF A PSYCHOSOCIAL CARE CENTER: A STUDY OF THEIR SOCIAL OUTCOMES ABOUT PSYCHIATRIC TREATMENT}

The purpose of this study is to identify and analyze representations users of a psychosocial care center construct about psychiatric treatment. Interviews were held with 11 individuals at a Psychosocial Care Center. Data were analyzed through content analysis, according to Bardin, using the Moscovician social representation theory as a methodological-theoretical reference framework. Data analysis allowed us to define three categories. The first category - treatment is to be medicatedcontains issues like medication as psychiatric treatment, the effects of treatment, various professionals involved and their participation in treatment. The second category - treatment is to be cared for (or not) - was outlined as: care in psychiatric treatment, violence and closed institutions. The third category - treatment is to be active- was based on: treatment and occupational therapy, psychiatric treatment and work. We concluded that the representations about psychiatric treatment are closely linked to the use of medication and to the physician as the precursor of the treatment.

DESCRIPTORS: mental health; social psychology, social; nursing; deinstitutionalization

\section{USUARIOS DE UN CENTRO DE ATENCIÓN PSICOSOCIAL: UN ESTUDIO DE SUS REPRESENTACIONES SOCIALES ACERCA DEL TRATAMIENTO PSIQUIATRICO}

La finalidad de este estudio es la identificación y análisis de las representaciones que los usuarios del centro de atención sicológica y social elaboran acerca del fenómeno tratamiento psiquiatrico. Fueron entrevistados 11 usuarios del Centro de Atención - CAPS/Estação. Los datos fueron sometidos al análisis de contenido, según Bardin, teniendo como soporte referencial teorico-metodológico la teoría moscoviciana de representación social. Los temas, definidos a partir de los dados, posibilitaron la estructuración de tres categorías. La primera de ellas, tratar es ser medicado, en esta categoría están contenidos diferentes temas como el remedio como tratamiento psiquiátrico, los efectos del tratamiento, los diferentes profesionales y su participación en el tratamiento. La secunda categoría es ser cuidado/ descuidado, los temas presentes fueron: cuidado en el tratamiento psiquiátrico, violencia e institución cerrada. La tercera categoría, tratar es estar en actividad, fue constituida a partir de los temas: tratamiento y la actividad ocupacional, tratamiento psiquiátrico y trabajo. Se concluye que las representaciones del tratamiento psiquiátrico están fuertemente conectadas a la utilización del medicamento y al médico, como precursor del tratamiento.

DESCRIPTORES: salud mental; psicologia social; enfermería; desinstitucionalizacion

\footnotetext{
${ }^{1}$ Psicóloga, Mestre pelo Departamento de Enfermagem da Faculdade de Ciências Médicas da Universidade Estadual de Campinas, e-mail: mostazo@bol.com.br; ${ }^{2}$ Enfermeira, Professor Assistente Doutor da Faculdade de Ciências Médicas da Universidade Estadual de Campinas
} 
As constantes queixas com relação ao tratamento psiquiátrico hospitalar por parte de indivíduos portadores de transtornos mentais internados em um hospital psiquiátrico, no qual a autora atuava com psicóloga, permitiram o questionamento de como seria a representação de tratamento psiquiátrico para o usuário de um programa extra-hospitalar de atendimento à saúde mental.

Acredita-se que o melhor entendimento da forma como estas pessoas compreendem a sua inserção em um programa extra-hospitalar possibilitará identificar elementos que possam orientar a busca de intervenções que objetive a maior adesão ao cuidado em saúde mental.

Entende-se, porém, que o sujeito constrói significados para o tratamento psiquiátrico de acordo com os sentidos ou símbolos atribuídos pela história social ${ }^{(1)}$. Dessa forma, optou-se por iniciar esse artigo com breve retrospectiva histórica e social das formas de tratamento do adoecimento psíquico, que vem se consolidando no decorrer dos séculos como conhecimento e prática na assistência ao portador de transtorno mental.

Até a Idade Média, a loucura era praticamente despercebida como doença e, quando notada, era vista como fato cotidiano ou como dádiva divina, por meio de significações religiosas e mágicas. A loucura tinha na sociedade uma certa razão, um ingrediente natural que habitava as casas, os povoados e os castelos $^{(2)}$. Os insanos, assim como os portadores de deficiência mental e os miseráveis, eram considerados parte da sociedade e o principal alvo da caridade dos mais abastados, que assim procuravam se redimir dos seus pecados. Apenas os casos mais extremados ou perigosos sofriam segregação social, e as recomendações terapêuticas eram: o contato com a natureza, viagens, repouso, passeio, retiro e o teatro, no qual se apresentava ao louco a comédia de sua própria loucura ${ }^{(3)}$.

No final do século XVIII, acontecimentos históricos repercutiram, na Europa Ocidental, idéias liberais e libertadoras, passando a se estabelecer como discurso, principalmente na França, onde havia clima de luta pelos direitos de cidadania e de valorização do homem. Esse movimento revolucionário fez com que o jovem médico Phillipe Pinel ficasse conhecido como aquele que libertou os loucos de suas correntes e os inseriu definitivamente no universo dos enfermos ${ }^{(4)}$. século XVII, perdeu sua força no final do século XIX. A psiquiatria assumiria posição positivista, centrada na medicina biológica.

Com os avanços no campo da medicina, a psiquiatria se reestabelece por meio do modelo biomédico no tratamento das doenças mentais. O modelo biomédico é resultado da influência do paradigma cartesiano no qual o corpo é identificado como uma máquina que pode ser analisada pelo estudo de suas partes ${ }^{(5)}$.

Na segunda metade do século XX, a quimioterapia tornou-se importante área de pesquisa e prática do tratamento para a doença mental. Em 1949, foi descrito o tratamento da excitação maníaca com o lítio, considerado momento importante na história da psicofarmacologia, que passa a ser o início de um processo farmacológico no tratamento da doença mental ${ }^{(6)}$.

No Brasil, até a segunda metade do século XIX, não houve assistência médica específica aos doentes mentais. A inauguração do primeiro hospital psiquiátrico no Brasil ocorreu em 1852, no Rio de Janeiro. Ele recebeu o nome Hospício D. Pedro II, e foi dirigido pelos religiosos da Santa Casa de Misericórdia. Em 1886, um médico psiquiatra, pela primeira vez, ocupou a direção do Hospício $^{(7)}$.

O tratamento atribuído aos seus internos tinha como objetivo suprimir a loucura. Para isso, alguns princípios deveriam organizar o espaço e a vida asilar. $\mathrm{O}$ isolamento e a vigilância tornaram-se princípios primordiais no tratamento e na segurança dos alienados. A condição de excluir o alienado da sociedade implicava a possibilidade de uma reinserção futura, depois de sua reabilitação através do tratamento oferecido pelo hospício ${ }^{(8)}$.

Na segunda metade da década de 80 , ocorreu no Brasil a implantação do processo de Reforma Psiquiátrica, que se consolidou norteada por acontecimentos marcantes, como o II Encontro Nacional dos Trabalhadores de Saúde Mental, em Bauru, SP, induzindo novos rumos para a saúde mental. Assim, a preocupação deixou de estar centralizada apenas na instituição psiquiátrica e passou a abranger a condição humana, social, política e cultural do doente mental, abrindo espaço para um questionamento viável e crítico ${ }^{(9)}$.

Dentre as várias experiências que emergiram ao longo da década de 90, com o propósito de implementar as diretrizes propostas pela Reforma Psiquiátrica Brasileira, 
destacou-se o processo de transformação do modelo assistencial percorrido pelo Serviço de Saúde Dr. Cândido Ferreira (SSCF), localizado em Campinas, SP. Desde 1991, por meio de suas ações de desinstitucionalização e de reabilitação psicossocial, intenciona superar a utilização da internação psiquiátrica como forma exclusiva de tratamento para a doença mental. Esse serviço tem por objetivo possibilitar a emergência no aparecimento de outras vertentes e modalidades de cuidados, visando introduzir os princípios que conduziram à implementação da Reforma Psiquiátrica em Campinas.

O Centro de Atenção Psicossocial CAPSEstação, integrou-se entre as Unidades pertencentes ao SSCF, sendo inaugurado em abril de 2000, localizado na região central de Campinas, esse serviço presta atendimento aos indivíduos com transtornos mentais, encaminhados pelas Unidades Básicas de Saúde, e também pelo próprio SSCF.

A estratégia de base do modelo de assistência do SSCF se constituiu por meio de dois pontos principais: a desospitalização e a criação de ações de reabilitação psicossocial. O Centro de Atenção Psicossocial-CAPS/ Estação, como Unidade desse Serviço, integrou essa concepção reabilitadora.

O intuito desta investigação limita-se a compreender como o fenômeno tratamento psiquiátrico é elaborado por indivíduos com histórico psiquiátrico vinculado ao Centro de Atenção Psicossocial-CAPS/Estação, partindo do pressuposto de que as ações dos indivíduos são orientadas pelas representações estabelecidas no seu cotidiano.

\section{OBJETIVO}

- Identificar e analisar as representações acerca de tratamento psiquiátrico, elaboradas por usuários de um Centro de Atenção Psicossocial.

\section{PERCURSO METODOLÓGICO}

Este estudo aproximou-se da perspectiva teórica do conceito de representação social, tendo como principais autores Serge Moscovici e Denise Jodelet.
A representação social foi abordada como modalidade de conhecimento particular que tem por função a elaboração de comportamentos e a comunicação entre os indivíduos ${ }^{(1)}$.

A noção de representação social se constitui na maneira de interpretar e de pensar a realidade cotidiana, ou seja, uma forma de conhecimento social ${ }^{(10)}$.

O estudo foi realizado no Centro de Atenção Psicossocial (CAPS)-Estação, unidade pertencente ao Serviço de Saúde Dr. Cândido Ferreira.

Participou da presente pesquisa grupo composto por 11 usuários do CAPS-Estação, de ambos os sexos, e que atendiam aos seguintes critérios: 1 . estar inserido no programa de atendimento do CAPS-Estação; 2. estar em condições físicas e psíquicas* de participar das entrevistas e 3. haver consentimento tanto do paciente quanto de seu responsável.

O presente estudo teve aprovação do Comitê de Ética em Pesquisa da Faculdade de Ciências Médicas da Unicamp e do Comitê de Ética do Serviço de Saúde Dr. Cândido Ferreira.

Foi também estabelecido que, na divulgação dos resultados, os nomes dos sujeitos seriam omitidos e substituídos por nomes fictícios.

As entrevistas foram realizadas individualmente pela pesquisadora, seguindo o modelo de entrevista semiestruturada. Organizou-se um roteiro com as principais questões abordadas na entrevista.

Optou-se por trabalhar com o material obtido através da análise do conteúdo ${ }^{(11)}$. A técnica de análise de dados utilizada na análise do conteúdo foi a da análise temática, considerando uma das formas que melhor se adequa à investigação qualitativa das pesquisas em saúde ${ }^{(12)}$.

\section{APRESENTAÇÃO E DISCUSSÃO DOS RESULTADOS}

A partir da análise das entrevistas foi possível organizar os temas em fundamentalmente três categorias temáticas: "tratar é ser medicado", "tratar é ser cuidado/(des)cuidado", "tratar é estar em atividade".

O "tratar é ser medicado" foi estabelecido como a primeira categoria, e está relacionada a temas que

* Foram excluídos os usuários em crise, como critério para a entrevista 
refletem a influência dos medicamentos, a participação dos profissionais e os efeitos esperados no tratamento psiquiátrico, segundo as representações dos entrevistados.

A demanda de medicamento mantém-se fincada em complexo sistema de idéias, expectativas e representações acerca do tratamento. A propaganda da indústria farmacológica se estabelece de forma ostensiva, assegurando a idéia de que tratar é sinônimo de medicar ${ }^{(9)}$. O que se pode constatar no depoimento a seguir - GETÚLIO: O meu tratamento psiquiátrico no hospital era tomar remédio, porque o tratamento me fazia bem. Os remédios me faziam bem (...) 0 tratamento psiquiátrico vai bem, a gente toma remédio corretamente.

A terapia medicamentosa dos transtornos mentais pode ser definida como tentativas de modificar ou corrigir comportamentos, humores ou pensamentos patológicos pela química ${ }^{(6)}$.

Dessa forma, o que se pode perceber é que o uso dos medicamentos no tratamento psiquiátrico se mantém por meio de uma representação socialmente compartilhada, permitindo, assim, adesão significativa da medicação como condição básica de tratamento psiquiátrico.

No entanto, quando os usuários se referem aos profissionais e às formas de tratamento, eles se limitam a apontar o médico, o enfermeiro e o psicólogo, ainda que a equipe seja composta também por outros profissionais. GETÚlIO: No postinho, eu parei o tratamento, pois faltava médico. Só tinha um médico lá. OLINDA: Meu tratamento aqui é bom. Tem médico que é muito legal, né?

No século XIX, todas as medidas, ações e gestos realizados no hospício de alienados tinham alcance terapêutico, devendo estar subordinados à decisão do médico ${ }^{(13)}$. Era fundamental que o poder e a informação no tratamento moral fossem concentrados na figura do médico.

O enfermeiro e o psicólogo foram identificados pelos entrevistados enquanto membros da equipe que institui o tratamento psiquiátrico, mas com papéis secundários àqueles do médico. A própria seqüência de citações dos profissionais na fala do entrevistado explicita essa questão - OSMAR: Com relação ao tratamento psiquiátrico, eu tô fazendo meu tratamento com a Dra Márcia e com a psicóloga e os enfermeiros.

No que se refere à definição dos papéis de cada profissional, sobre a enfermagem, são primordialmente citados os aspectos de execução de ordem médica. Embora o cuidado seja a função específica desse profissional, nas falas dos sujeitos o enfermeiro tem sua competência associada às qualidades pessoais. Dessa forma, de acordo com os sujeitos entrevistados, o enfermeiro competente é aquele "que dá remédio" e é "muito bonzinho".

Vê-se que tal representação pode estar relacionada com a visão que se conformou no senso comum, com base num conhecimento produzido pelos próprios pioneiros da psiquiatria, pois, na primeira metade do século $\mathrm{XX}$, nos hospícios brasileiros, os psiquiatras desejavam que a enfermagem se realizasse a partir de aptidão e vocação. Julgavam fundamentais para o perfil dos trabalhadores de enfermagem, que assistiam os doentes mentais, a paciência e a doçura, intercaladas com energia e firmeza de caráter ${ }^{(14)}$.

Observa-se que, para os entrevistados, a equipe se centraliza na figura do médico, apesar deles reconhecerem o enfermeiro e o psicólogo. Eles sempre se reportam ao médico por ser aquele que prescreve a medicação, e enunciam a enfermagem como aquela responsável pelo cuidado. Nas falas dos entrevistados, não há diferenciação entre o auxiliar de enfermagem e o enfermeiro, uma vez que a identificação profissional é descrita de forma generalizada. Ao psicólogo é atribuída a característica profissional de ouvir e de conversar.

Em relação às representações que os entrevistados apresentam sobre os efeitos ou perspectivas do tratamento psiquiátrico, foi percebida a crença na cura. Para eles, a cura significa a não necessidade de medicação, o retorno ao convívio familiar ou a conquista do próprio espaço de moradia e de trabalho. MARILDA: Eu gostaria de já estar boa. Faz um ano que eu já tô aqui me tratando. Eu queria estar boa já, não tendo mais nada, né?

No século XIX, a prática objetivava a cura para com os insanos como característica central da medicina mental. Os psiquiatras divulgavam a necessidade de um tratamento para os loucos, resultando em atitude ativa, transformadora de sintomas e de alterações morais. Nesse sentido, foi instaurada a prática da cura por meio do Tratamento Moral, que se tornou o procedimento nuclear para a cura dos alienados ${ }^{(13)}$. Nota-se que, até a primeira metade do século $\mathrm{XX}$, a eficácia do hospício para a sociedade residia na operacionalidade da cura.

O que se pode depreender nos depoimentos é que, para os entrevistados, a representação da cura se mantém possível por meio do tratamento. Em suma, a possibilidade de cura com o tratamento psiquiátrico se ancora no tratamento moral, ao qual foi destinada essa função e que se codificou na representação dos 
entrevistados.

A segunda categoria "tratar é ser cuidado/ (des)cuidado" foi estruturada a partir dos temas: o cuidado no tratamento psiquiátrico e violência e instituição fechada, uma vez que esses temas indicam as ações de cuidado e descuido atribuídas ao tratamento psiquiátrico pelos sujeitos entrevistados.

O cuidado no tratamento psiquiátrico é percebido pelos usuários como a obtenção da medicação e também como suporte nas atividades da vida prática. Tal qual a medicação, as atividades da vida prática também são mencionadas como cuidados decorrentes do tratamento psiquiátrico. ALCEU: O tratamento psiquiátrico aqui no CAPS é bom. A gente come uma comida muito boa. O tratamento está sendo excelente, porque eu tô sendo bem cuidado pela doutora. Todos os pacientes são bem cuidados.

A partir do momento em que a psiquiatria teve início e a loucura passou a ser considerada uma enfermidade, portanto, a concepção de que a loucura deveria ser cuidada por outras pessoas tornou-se predominante na história psiquiátrica ${ }^{(15)}$.

$\mathrm{O}$ isolamento, a falta de liberdade e a falta de higiene se mantêm ainda como marco no cuidado psiquiátrico institucional, compatível com o modelo manicomial, como se depreende do que é dito pelos entrevistados. Como se pode identificar no depoimento abaixo descrito - ANA: Eu cheguei a agredir a enfermeira em um dos hospitais que eu estive, e daí eu usei camisa de força ... Todas as portas eram fechadas, chaveadas, num espaço aberto ao relento, com chuva e com sol, no tempo que fosse. As pessoas tiravam as roupas, um ambiente horrível, pois as pessoas faziam cocô. Chegaram a comer o cocô na minha frente ... Foi horrível.

A reclusão de pessoas portadoras de transtornos mentais teve, no curso da história, várias modalidades. A mais antiga era de recolhê-los junto com outras minorias sociais, desempenhando, assim, a função de abrigo ou recolhimento. Posteriormente, apareceram as instituições hospitalares que destinavam espaços ou edifícios inteiros exclusivamente ao recolhimento de alienados. Nas primeiras décadas do século XIX, o acolhimento aos doentes mentais se caracterizou pelo tratamento médico sistemático e especializado. As condições de vida dos alienados nos hospícios eram de reclusão e condições sanitárias extremamente precárias e sob uma disciplina brutal $^{(16)}$.

A terceira categoria "tratar é estar em atividade" se estrutura pela representação que os entrevistados atribuem à atividade ocupacional e ao trabalho no tratamento psiquiátrico, possibilitando a reinserção social.

Para os entrevistados, as atividades ocupacionais constituem-se na retomada da autonomia e das atividades da vida diária. Os usuários percebem as suas potencialidades e o êxito quando desenvolvem atividades, seja na terapia ocupacional quanto na vida cotidiana. Uma das entrevistadas mostra-se auto-suficiente quando afirma sua autonomia na realização de determinadas atividades, como se pode identificar no relato descrito a seguir MARILDA: Eu gosto de lavar louça, fazer barrinha no pano, fazer limpeza, fazer comida, ajudar em um monte de coisa. O que eu faço na minha casa, é lavar, passar, e tudo. Eu sou uma pessoa suficiente para isso, graças a Deus (...) Meu cabelo? Eu arrumo meu cabelo do gosto que eu quero. Eu chego com uma roupa bem limpinha. Às vezes tem gente que pergunta quem é que lava minha roupa, sou eu mesma! É, eu mesma que lavo! (...) Sobre o tratamento, eu tô me virando com a pintura e outras coisas, como uma barrinha que eu faço. É pra mim não estar esperando a médica, porque é muita coisa para a cabeça dela.

As atividades da vida diária se caracterizam pelos cuidados pessoais, em atividades realizadas pelo homem no seu cotidiano, tais como: alimentação, higiene pessoal, locomoção, comunicação, habilidades manuais e tarefas domésticas ${ }^{(17)}$.

Verifica-se que, para a entrevistada, a satisfação no cuidado das atividades da vida diária lhe proporciona um novo direcionamento para o seu tratamento psiquiátrico, com o reconhecimento de suas habilidades no seu próprio cuidado.

A noção de saúde mental se constitui de forma complexa, não podendo ser contida em um modelo exclusivamente biológico e médico, posto que implica ações múltiplas que envolvem o social e o cultural ${ }^{(18)}$

As atividades ocupacionais nas falas dos entrevistados perdem seu caráter terapêutico e avançam em uma representação de produção. O trabalho não apenas traz bem-estar, mas também a remuneração e a recolocação do usuário do CAPS no mercado de trabalho, o que é identificado na fala a seguir - MARILDA: Eu queria tá boa já, trabalhando aqui, com eles, fazendo uma atividade séria e trabalhando junto, né? Já não é uma atividade da TO, né? Já é uma outra atividade que nem a deles. Se eu já tivesse boa, eu já estaria trabalhando aqui, como funcionária, porque eu tenho esse sonho.

A idéia de saúde para a classe trabalhadora está fortemente ligada à chance de trabalhar. O corpo é efetivamente um instrumento de trabalho. Essa parece ser uma função primordial e que determina, por assim 
dizer, o que é doença ou não, na medida em que esteja ou não ameaçada ${ }^{(9)}$. Isso vai fazer com que a noção de saúde esteja bastante vinculada à possibilidade de retomada do trabalho.

As representações de trabalho no tratamento psiquiátrico são expressivas nos depoimentos, pois, para o entrevistado, a condição de estar inserido no mercado de trabalho é uma conseqüência da eficácia do tratamento psiquiátrico, que pode trazer a cura e, por conseguinte, proporcionar a possibilidade de trabalhar. A idéia de saúde está fortemente ligada à condição de trabalho, visto que o ser humano está bem quando se mantém apto para o trabalho ${ }^{(9)}$.

\section{CONSIDERAÇÕES FINAIS}

Este trabalho tinha como objetivo identificar e analisar as representações de tratamento psiquiátrico elaboradas por usuários de um centro de atenção psicossocial. Para alcançá-lo foi realizada pesquisa qualitativa. Com base nos dados coletados concluiu-se que as representações de tratamento psiquiátrico estão fortemente ligadas ao uso do medicamento e ao médico como precursor do tratamento. A esse profissional é dada a responsabilidade de medicar.

A equipe de enfermagem é representada pela função de cuidador, e ao psicólogo é destinado o papel de ouvir e conversar. Instituições fechadas e violência no tratamento são apontadas nos depoimentos como fatores presentes na representação de tratamento psiquiátrico.

As atividades ocupacionais e o trabalho se perpetuam por meio de relações interpessoais, possibilitando aos sujeitos maior perspectiva na interlocução social, reintegrando o indivíduo portador de transtorno mental ao seu meio.

14. Kirschbaum DIR. Análise histórica das práticas de enfermagem no campo da assistência psiquiátrica no Brasil, no período compreendido entre as décadas de 20 e 50 . Rev Latino-am enfermagem 1997 maio; 5( $\mathrm{n}^{\circ}$ especial):19-30. 15. Szasz ST. O mito da doença mental. São Paulo (SP): Círculo do Livro; 1974

16. Pessotti I. O século dos manicômios. São Paulo (SP): Ed. 34; 1996.

17. Francisco RB. Terapia Ocupacional. $2^{\mathrm{a}}$ ed. Campinas (SP): Papirus; 2001

18. Saraceno B. Libertando identidades. Da reabilitação psicossociais à cidadania possível. Rio de Janeiro (RJ): Te Corá; 1999. 